\title{
Revisão de Literatura
}

\section{Entrevista motivacional e escalas de motivação para tratamento em dependência de drogas Motivational Interview and scales used to assess the degree of motivation for treatment in drug abuse patients}

\author{
Marcelle M. Lobo Dinis Castro ${ }^{1}$ \\ SôNia Regina LAMBERT Passos ${ }^{2}$
}

\begin{abstract}
Resumo
Objetivo: Identificar e rever os instrumentos que explorem a motivação como fator capaz de predizer o resultado de tratamento da dependência de substâncias psicoativas e que vêm sendo utilizados na última década. Método: Revisão bibliográfica abrangente de literatura científica indexada sobre escalas que aferem o grau motivação. Resultado: Identificaramse quatro instrumentos e suas propriedades psicométricas, os dois primeiros com versões validadas em português: Rhode Island Change Assessment Questionnaire (URICA), Stages of Change Readiness and Treatment Eargness Scale (SOCRATES), Treatment Motivation Questionnaire (TMQ), Readiness to Change Questionnaire (RCQ). Conclusão: A teoria motivacional vem sendo regularmente estudada na última década, permitindo avaliação pragmática de seus parâmetros por meio de escalas com validade e confiabilidades que variam de boa a excelente.
\end{abstract}

Palavras-chaves: Dependência de drogas, motivação, entrevista motivacional, escalas.

\begin{abstract}
Objective: To identify and review tools used in specialized services to explore aspectos of motivation as a predictive factor of treatment outcome in drug-abusing patients. Method: Comprehensive literature review of scales used to assess the degree of motivation. Result: Four scales to evaluate stages of behavioural change related to motivation were identified and described, together with their associated psychometric properties - Rhode Island Change Assessment Questionnaire (URICA), Stages of Change Readiness and Treatment Eargness Scale (SOCRATES), Treatment Motivation Questionnaire (TMQ), Readiness to Change Questionnaire (RCQ); The first two have Portuguese validated versions. Conclusion: Motivacional theory has been regularly studied in the last decade, allowing the definition of pragmatic evaluation parameters in scales with validity and reliabilities that vary from good to excellent.
\end{abstract}

Key-words: Drug dependence, motivation, motivational interview, scales.

1 Médica Psiquiatra. Aluna do Curso de Especialização em Assistência a Dependentes de Álcool e outras Drogas do Núcleo de Estudos e Pesquisa em Atenção ao Uso de Drogas (NEPAD) da Universidade do Estado do Rio de Janeiro (UERJ).

2 Pesquisadora do Departamento de Epidemiologia e Antropologia do Instituto de Pesquisa Clínica Evandro Chagas da Fundação Oswaldo Cruz (IPEC /FIOCRUZ). Médica psiquiatra do Núcleo de Estudos e Pesquisas em Atenção Uso de Drogas (NEPAD - UERJ). Doutora em Saúde Pública.

Endereço para correspondência: Fundação Oswaldo Cruz (FIOCRUZ) Departamento de Epidemiologia e Antropologia do Instituto de Pesquisa Clínica Evandro Chagas. Av. Brasil, 4365, Manguinhos - 21045-900

- Rio de Janeiro - RJ. E-mail: lobodinis@ig.com.br 


\section{Introdução}

A motivação caracteriza-se como processo dinâmico segundo o modelo transteórico, desenvolvido por Prochasca e Diclement (1983). Este modelo descreve os estágios de mudança comportamental por meio dos quais o indivíduo "transita” de forma não linear, estando em tratamento ou não. Tais estágios são: pré-contemplação, contemplação, determinação, ação, manutenção e recaída.

Um aspecto relevante é a origem da influência da motivação que pode ser externa (pressões, ações coercitivas) ou interna (motivação que vem do próprio indivíduo) (Ryan e Plant, 1995).

Há controvérsias sobre a repercussão da maior gravidade da dependência de substâncias psicoativas e sua correlação com os níveis de motivação, que tanto podem aumentar a chance de recaída em pacientes ambulatoriais e internados (Silva et al., 1995; Franken e Hendricks, 1999), quanto pode diminuir a probabilidade de abandono de tratamento (Ryan e Plant, 1995; Carpenter et al., 2002).

As comorbidades, também, podem influenciar a resposta ao tratamento da dependência por alterar a motivação. A depressão, por exemplo, pode interferir na motivação para a abstinência de álcool e de outras drogas. Alcoolistas com sintomas depressivos graves mostravam-se mais motivados para uma mudança comportamental, provavelmente, por possuírem maior percepção das conseqüências negativas da dependência do álcool (Blume et al., 2001). Cahill et al. (2003) encontraram uma correlação forte e direta entre depressão e motivação interna $(\mathrm{p}<0,002)$, motivação externa $(\mathrm{p}<$ $0,01)$ e a busca de ajuda interpessoal $(\mathrm{p}<0,01)$.

A entrevista motivacional (EM) é uma abordagem criada para auxiliar o sujeito a reconhecer seus problemas atuais e potenciais quando há ambivalência quanto à mudança comportamental e estimular o comprometimento para a realização dessa mudança por meio de abordagem psicoterápica persuasiva e encorajadora (Miller e Rollnick, 2001).

Esta técnica propõe intervenções terapêuticas individualizadas adequadas a cada estágio com vistas a aumentar a adesão ao tratamento e prevenir possíveis recaídas em pacientes com comportamentos considerados dependentes, transtornos alimentares, tabagismo, jogo patológico, dependência de substâncias psicoativas, e, também, em comportamentos sadios com vistas à promoção de saúde (Miller e Rollnick, 2001; Oliveira e Malbergier, 2003).

A intervenção motivacional breve (IMB) tem importante funcionalidade por ser uma forma de atendimento concisa, baseada na EM, que objetiva alcançar a mudança comportamental do paciente e desencadear uma tomada de decisão e o comprometimento com a mudança (Miller e Rollnick, 2001). Neste tipo de intervenção, utiliza-se um inventário de estratégias oferecidas pelo entrevistador, ao paciente, para modificar seu comportamento problema de modo responsável.

A IMB consiste em uma a três sessões, que possuem impacto motivacional e precipitam mudanças de comportamento, sendo comparável a tratamentos mais extensos em dependência de drogas. Ela contém seis elementos: devolução; responsabilidade pessoal do paciente; conselhos claros para mudança de hábito; seleção de uma abordagem específica de tratamento, mas ofertando estratégias alternativas; empatia do terapeuta; e reforço da auto-eficácia da esperança do paciente (Millner e Rollnick, 2001).

A atuação do terapeuta ao conduzir uma IMB pauta-se em cinco princípios estruturantes: promoção da auto-eficácia, expressão de empatia, desenvolvimento de discrepância, evitação de argumentação e acompanhamento da resistência.

Auto-eficácia é a crença de uma pessoa em sua capacidade de realizar ou de ter êxito em uma tarefa específica. Ela pode ser considerada como elementochave na motivação para mudança e bom indicador de resultados de tratamento (Miller e Rollnick, 2001). De Weert-van Oene et al. (2001) justificam a auto-eficácia como bom indicador quanto à duração do tratamento. Pacientes com maior auto-eficácia apresentam maior capacidade de enfrentar seus problemas relacionados à dependência de substâncias psicoativas e, por isso, alcançam desfecho do tratamento mais rapidamente.

Expressar empatia é a capacidade de construção de aliança terapêutica conseguida pela escuta reflexiva e respeitosa e o desejo de compreender as esperanças e os receios do paciente. A "aceitação" dos limites do paciente facilita a ocorrência para mudança comportamental.

Desenvolver discrepância consiste em ajudar o paciente a conscientizar-se dos custos de seu comportamento atual, relacionados à dependência de drogas, e de seus recursos pessoais para reformular seus projetos de vida. Blume et al. (2001) afirmam que quando pacientes dependentes de álcool conscientizam-se que os custos de sua dependência sobrepõem-se aos benefícios e prazeres da bebida, ficam mais motivados em mudar seu comportamento. Perdas frequientes relacionadas às substâncias psicoativas podem ser fatores preditivos para aumentar os níveis de motivação pessoal para mudança comportamental (Blume e Marlatt, 2000; Blume e Schmaling,1998).

Evitar a argumentação significa evitar discussões contraproducentes acerca do diagnóstico de dependência de substâncias psicoativas.

Acompanhar a resistência caracteriza-se por reconhecer o estado motivacional do paciente e pautar as intervenções terapêuticas individuais. Escalas que aferem o estágio de motivação do paciente têm sido construídas, na tentativa de aumentar a precisão e validade da identificação do estágio atual de um paciente em particular e auxiliar o terapeuta na escolha da estratégia de tratamento mais adequada. 
Este artigo objetiva identificar e revisar os instrumentos que exploram a motivação como fator capaz de predizer o resultado de tratamento da dependência de substâncias psicoativas e subsidiar a conduta terapêutica.

\section{Método}

A realização deste trabalho consistiu de revisão bibliográfica abrangente de artigos nacionais e internacionais, que compreendeu o período de 1995 a 2004, publicados em revistas indexadas no MedLine, Lilacs e Embase, Scielo, Psyclite nas áreas de toxicomania, psiquiatria e psicologia utilizando as palavras-chaves: "dependência de drogas, motivação, entrevista motivacional e escalas de avaliação", além de livros especializados.

\section{Resultados}

\section{Escalas de motivação para tratamento em dependência de drogas}

Diversos estudos têm utilizado uma série de instrumentos que avaliam o aspecto motivacional de mudança comportamental, entre eles: Rhode Island Change Assessment Questionnaire (URICA), Stages of Change Readiness and Treatment Eargness Scale (SOCRATES), Treatment Motivation Questionnaire (TMQ), Readiness to Change Questionnaire (RCQ). O número de artigos identificado por instrumento foi: URICA (7), SOCRATES (6), RCQ (3) e TMQ (2). URICA e SOCRATES são de domínio público, não envolvem problemas de copyright e podem ser utilizadas sem custo.

URICA é um questionário de auto-preenchimento que não necessita de treinamento prévio, aplicado na população adulta, contém 32 perguntas divididas em quatro subescalas, as quais abrangem os seguintes estágios de mudança comportamental: pré-contemplação, contemplação, ação e manutenção. Há uma versão com 24 itens. O tempo necessário para a aplicação da escala varia de cinco a dez minutos. Este instrumento investiga os estágios motivacionais de pacientes que procuram tratamento para a modificação de qualquer tipo de problema comportamental e visa fornecer esta informação para ajudar a guiar as abordagens de tratamento adequadas. URICA encontra-se ainda em processo de validação em sua versão em português (Oliveira et al., 2003).

Oliveira et al. (2003) aplicaram o URICA e o Shortform Alchool Dependence Data (SADD) em uma amostra de pacientes alcoolistas em serviço especializado e observaram correlação positiva $(r=0,197 ; p=0,031)$ entre o estágio de manutenção e maior gravidade de dependência de álcool. A proposta de tratamento oferecida foi estimular a auto-eficácia destes pacientes como forma de prevenção à recaída.

URICA é considerado instrumento confiável e de boa consistência interna (Pantalon e Swanson, 2003) e validades concorrente e preditiva satisfatórias. Em uma amostra heterogênea de pacientes dependentes de álcool, a variância e a consistência interna da escala foi aceitável e variou de 0,8 a 0,84 e a validade preditiva foi encontrada em quatro dos cinco perfis replicados (Carney e Kivlahan, 1995). Velásquez et al. (1999) encontraram coeficientes de confiabilidade para as subescalas do URICA que oscilaram de 0,74 a 0,89 . O coeficiente alfa de Cronbach para a pontuação da subescala de prontidão foi de 0,91 .

Várias pesquisas têm sido realizadas utilizando o URICA a fim de estimar os estágios motivacionais em pacientes psiquiátricos com transtornos primários relativos ou não à dependência de drogas e/ou em comorbidades. Henderson et al. (2004) avaliaram a validade preditiva dos estágios de mudança em dependentes de heroína, como droga primária, usando o URICA para prever a adesão ao tratamento e amostras urinárias negativas de heroína e/ou cocaína durante um período de 29 semanas. Esses pacientes tiveram acesso a materiais didáticos sobre o assunto, participaram de terapias de grupo e cognitivo-comportamentais e de entrevistas motivacionais. A consistência interna das subescalas do URICA mostrou-se adequada e variou de 0,71 a 0,88 . O coeficiente alfa de Cronbach oscilou de 0,71 a 0,93 para o URICA relativo a amostras de cocaína urinária $\mathrm{e}$ 0,64 a 0,71 relativo a amostras de heroína na urina. As pontuações das subescalas da URICA resultaram em aumento significativo da variância capaz de predizer amostras urinárias negativas de heroína e cocaína e adesão ao tratamento. Após o controle das variáveis demográficas, da gravidade de uso e da escolha da terapêutica, a subescala de manutenção foi preditiva para a presença de amostras de urina livres de cocaína e para a adesão ao tratamento, enquanto a subescala de contemplação foi preditiva para a obtenção de amostras na urina livres de heroína.

SOCRATES, criada por William Miller, é um instrumento auto-administrável composto por 19 itens (versão recente), sendo as alternativas de resposta do tipo Likert, voltados para pacientes alcoolistas, com duração média de três minutos. Abrange três subescalas: reconhecimento (capacidade de insight do paciente com relação ao consumo de álcool); ambivalência e ação (evolução do paciente naquele período de avaliação). A subescala reconhecimento abrange itens que investigam a preparação e determinação, a subescala ambivalência engloba o item contemplação e a subescala ação inclui os itens ação e manutenção. Suas características psicométricas incluem boa confiabilidade e boa consistência interna (Miller e Tonigan,1996; Maisto et al., 1999; Maisto et al., 2003; Figlie et al., 2004).

Miller e Tonigan (1996) utilizaram SOCRATES para estimar a motivação de uma amostra de alcoolistas com altos níveis de gravidade e de consumo de bebida, identificaram que esta escala possuía uma estrutura de três fatores: ambivalência, reconhecimento e ação e é 
um instrumento de alta confiabilidade (o coeficiente de correlação intraclasse oscilou de 0,82 a 0,94).

Em estudo de validade concorrente, conduzido em uma amostra de pacientes alcoolistas graves de instituições de atendimento primário, Maisto et al. (1999) observaram que as subescalas de ambivalência e de reconhecimento (AMREC) estavam correlacionados aos níveis de gravidade do alcoolismo: as maiores pontuações da subescala AMREC estavam diretamente correlacionadas à frequiência e à quantidade dos problemas clínicos decorrentes do consumo alcoólico, e que a subescala ação (Taking steps) estava correlacionada à mudança comportamental, com as maiores pontuações relacionadas diretamente à redução de consumo alcoólico e posterior manutenção desta redução. Nos mesmos pacientes alcoolistas, após um período de seis meses de tratamento, a subescala AMREC demonstrou boa validade preditiva: a AMREC manteve a mesma correlação estatisticamente significativa com a gravidade e freqüência do consumo de álcool (expressa em número total e diário de drinques em período de seis meses), o que não ocorreu com subescala taking steps, que estava relacionada positivamente com a sintomatologia de abstinência, mas inversamente às conseqüências do consumo de bebidas alcoólicas.

Figlie et al. (2004) estudaram a consistência interna e a estrutura fatorial da versão em português da SOCRATES (versão 8) em uma amostra ambulatorial de um centro especializado em gastrenterologia de dependentes de álcool, baseando-se na estrutura fatorial explicitada por Miller e Tonigan (1996) e Maisto et al. (1999). No estudo de Fligie et al. (2004), SOCRATES apresentou boa consistência interna e confiabilidade. Similarmente ao trabalho de Maisto et al. (1999), Fligie et al. encontraram uma estrutura de dois fatores nãoindependentes agrupados ao redor de dois diferentes conceitos, o AMREC e o conceito de ação, talvez em virtude da semelhança de conceitos entre ambivalência e reconhecimento.

Uma outra escala disponível na literatura é a TMQ. Trata-se de instrumento auto-administrável, composto por 29 itens, que abrangem os motivos pelos quais o paciente procura e permanece no tratamento, distribuídos pela avaliação de quatro áreas: motivação interna, motivação externa, busca de ajuda interpessoal e grau de expectativa quanto ao resultado de tratamento (Cahill et al., 2003). Os itens variam em uma escala de cinco pontos, incluindo as opções "não é totalmente verdadeira" a "totalmente verdadeira". A TMQ apresenta boa validade de construto, por demonstrar alta interdependência entre as variáveis presentes na escala (Ryan e Plant, 1995).

Ryan e Plant (1995) aplicaram a TMQ em uma amostra de dependentes de álcool revelando que a motivação interna estava associada com a maior adesão ao tratamento, principalmente se estivesse associada à alta motivação externa.
Cahill et al. (2003), utilizando também a TMQ com o intuito de estimar o grau de motivação em uma amostra de pacientes do sexo masculino dependentes de álcool e drogas, observaram não haver diferença significativa entre as pontuações de motivação dos dependentes de drogas e de álcool anterior e posteriormente ao tratamento a que foram submetidos. Em ambos os grupos de pacientes, perceberam que as pontuações da motivação externa, da motivação interna e da motivação para a busca de ajuda interpessoal no pré-tratamento mantiveram-se altas após o término deste e que no decorrer do mesmo, somente a motivação externa mostrou-se em queda.

RCQ é um questionário composto por 12 itens, que abrangem os estágios de mudança motivacionais: précontemplação, contemplação e ação. O estágio de manutenção não é abordado por esse instrumento porque, para indivíduos abstêmios, muitas das perguntas, desse questionário, não fazem sentido e conseqüentemente o questionário pode apresentar um mau entendimento (Rumpf et al., 1999). Este questionário foi desenvolvido para avaliar pacientes dependentes de álcool. Os itens variam em uma escala de cinco pontos abrangendo opções "não concordo completamente" a "concordo completamente". Altas pontuações nas escalas de contemplação e ação e baixas pontuações na escala de pré-contemplação indicam maior prontidão para mudança comportamental.

A partir da proposta da referida escala, Rumpf $e t$ al. (1999) avaliaram os estágios de mudança por meio da aplicação da RCQ em duas amostras representativas de indivíduos dependentes de álcool de um hospital geral e da população geral. Observaram que os pacientes do hospital geral encontravam-se mais freqüentemente no estágio de ação $(\mathrm{p}<0,0001)$, ou seja, "prontos para mudança" de atitudes frente à dependência alcoólica, quando comparados aos alcoólicos da população geral.

Share et al. (2002) avaliaram a importância da análise custo-benefício (balança motivacional) no início do tratamento, a sua possível influência nos estágios motivacionais e na tomada de decisão com relação ao tratamento de mulheres dependentes de álcool. Ao correlacionar as pontuações da escala RCQ e a percepção custo-benefício (balança motivacional), observaram que as mulheres com maiores pontuações no estágio de pré-contemplação não se mostravam "capazes" de avaliar a relação custo-benefício de suas atitudes de dependência, apresentando menor diferença na relação custo-benefício. Por outro lado, pacientes que apresentavam maiores pontuações no estágio de ação, percebiam com menor intensidade os custos da mudança em seu comportamento alcoólico e apresentavam maior diferença na relação custo-benefício. Pacientes em estágio de contemplação não foram correlacionadas com as variáveis da balança motivacional, embora percebessem melhor os benefícios de mudança da sua atitude com relação à dependência alcoólica, quando comparadas às em estágio de pré-contemplação. 
Defuentes-Merillas et al. (2002) avaliaram as propriedades psicométricas da RCQ_D (versão alemã) em duas amostras de pacientes: uma de pacientes dependentes de álcool e/ou drogas em tratamento em centro especializado e a outra de pacientes alcoólicos infratores provenientes da justiça.

A estrutura fatorial da RCQ_D nessas duas amostras foi consistente com a estrutura fatorial da RCQ original britânica. A consistência interna foi satisfatória nos estágios: pré-contemplação $(\alpha 0,68)$; contemplação $(\alpha$ $0,7)$ e ação $(\alpha 0,81)$. Os estágios de mudança adjacentes mostraram maior intercorrelação quando comparados aos estágios não adjacentes. Como esperado, a amostra de pacientes dependentes em tratamento encontrava-se majoritariamente no estágio de ação.

A estrutura fatorial da RCQ_D diferiu ligeiramente da estrutura fatorial da RCQ original, revelando duas estruturas fatoriais: uma, bi-dimensional, responsável pelo agrupamento dos itens referentes à pré-contemplação e a contemplação; e a outra, unidimensional, responsável pelo agrupamento dos itens referentes à ação.

$O$ coeficiente $\alpha$ dos itens positivamente formulados referentes à pré-contemplação foi pouco maior $(\alpha$ $0,83)$ comparativamente aos itens negativos $(\alpha 0,81)$.

\section{Conclusão}

Identificou-se quatro escalas com parâmetros psicométricos variando de bom a excelente, todas elas em con- dições de serem utilizadas para avaliação de tratamento e predição de adesão ao mesmo. Pelo menos duas delas (URICA e SOCRATES) já possuem versão em português com validade e confiabilidade descritas.

O conceito de estágios motivacionais, que foi sedimentado no início da década de 1980, serviu de base para a construção de uma proposta de intervenção terapêutica (Entrevista motivacional) bem estabelecida e que vem sendo testada, internacionalmente, há pelo menos uma década e recentemente, também, no Brasil.

Com base no exposto, preconiza-se a prática de intervenções breves, por não-especialistas em dependência de drogas, tanto em consultas de clínica geral, quanto em adolescentes, com o intuito de despertar ou aumentar a motivação para a abstinência (Albernaz e Passos, 2001). A intervenção breve em adolescentes usuários de substâncias psicoativas foi mais efetiva em reduzir a prevalência do consumo e os comportamentos de risco associados a este, comparado a uma orientação preventiva contendo informações em folhetos (De Micheli et al., 2004).

Futuros desdobramentos deste trabalho incluem, em curto prazo, a avaliação da capacidade preditiva da URICA, a partir de sua utilização em estudo ambulatorial em centros de tratamento para dependentes de droga no Rio de Janeiro e, em médio prazo, viabilizar versões em português das escalas ainda não disponíveis (RCQ e TMQ).

\section{Referências bibliográficas}

Albernaz, A.L. G.; PAssos, S.R.L. - Uso de substâncias psicoativas. In: Coutinho, M.F.G.; Barros, R.R. (Org.) Adolescência: Uma abordagem prática. São Paulo: Atheneu, 2001.

Blume, A.W.; MARLATt, G.A. - Recent important substance-related losses predict readiness to change scores among people with co-occurring psychiatric disorders. Addict Behav 25(3):461-4, 2000.

Blume, A.W.; SchAMALING, K.B. - Regret substance abuse and readiness to change in dually diagnosed sample. Addict Behav 23(5):693-7, 1998.

Blume, A.W.; Schmaling, K.B.; Marlatt, G.A. - Motivation drinking behavior change depressive symptoms may not be noxious. Addict Behav 26:267-72, 2001.

Cahill, M.; Adinoff, B.; Hosig, H.; Muller, K.; Pulliam, C. - Motivation for treatment preceding and following a substance abuse program. Addict Behav 28:67-79, 2003.
Carney, M.M.; Kivlahan, D.R. - Motivational subtipes among veterans seeking substance abuse treatment profiles based on stages of change. Psycology of Addict Behav 9(2):135-42, 1995.

Carpenter, K.M.; Miele, M.M.; Hasin, D.S. - Does motivation to change mediate of DSM-IV substance use disorders on treatment utilization and substance abuse? Addict Behav 27:207-25, 2002.

De Michel, D.; FISBerg, M.; Formigoni, M.L.O.S. - Estudo da efetividade da Intervenção Breve para o uso de Álcool e outras drogas em adolescentes atendidos num serviço de assistência primária à saúde. $\operatorname{Rev} A M B$ 50(3):305-13, 2004.

De Weert-Van Oene, G.H.; Schippers, M.G.; De Jong C.A.J.; SCHRIJVERS, G.J.P. - Retention in substance dependence treatment the relevance of in-treatment factors. Journal of Substance Abuse Treatment 20:253-61, 2001. 
Defuentes-Merillas, L.; Dejong, C.A.J.; SchipPeRs, G.M. - Reliability and validity of the Dutch version of the Readiness to change Questionnaire. Alcohol and Alcholism 37(1):93-9, 2002.

Figlie, N.B.; Dunn J.; Laranjeira, R. - Estrutura Fatorial da Stages of Readiness and Treatment Eagerness Scale (Socrates) em dependentes de álcool tratados ambulatorialmente. Rev Bras Psiq 26(2):91-107, 2004.

Franken, I.H.A.; HendRIKS, V.M. - Predicting outcome of inpatient detoxication df substance abusers. Psychiatric Services 50:813-7, 1999.

Henderson, M.J.; Saules K.K.; Gale L.W. - The predictive validity of the University of Rhode Island Change Assessment Questionnaire in a Heroin-Addicted polysubstance abuse sample. Psych Addict Behav 18(2):106-12, 2004.

Maisto, S.A.; Chung, T.A.; Corneluus, J.R.; Martin, C.S. - Factor structure of the Socrates in a clinical sample of adolescents. Psychology of Addictive Behaviors 17(2):98-107, 2003.

Maisto, S.A.; Coniglaro, J.; Mcnell, M.; Kramer, K.; O’Connor, M.; KELLEY, M.E. - Factor structure of the Socrates in a sample of primary care patients. Addict Behav 24(6):879-92, 1999.

Mılıer, W.R., Rollnick, S. - Entrevista Motivacional: Preparando as pessoas para a mudança de comportamentos adictivos. Porto Alegre: Artes Médicas, 2001.

MılLer, W.R.; TonIGAN, J.S. - Assessing drinkers` motivation for change: The stages of Change Readiness and Treatment Eagerness Scale (Socrates). Psychology of Addict Behav 10(2):81-9, 1996.

Oliveira JR., H.P.; MalbergieR, A. - Assessment of motivation for treatment in alcohol dependent patients who sought treatment at a specialized medical service. Rev Bras Psiq 25(1):265-70, 2003.
Oliveira, M.; Laranjeira, R.; Araújo, R.; Camilo, R.; Schneider, D.D. - Estudo dos estágios motivacionais em sujeitos adultos dependentes de álcool. Psicologia: Reflexão $e$ Crítica 16(2):265-70, 2003.

Pantalon, M.V.; Swanson, A.J. - Use of the University of Rhode Island Change Assessment to measure motivational readiness to change in psychiatric and dually diagnosed individuals. Psychol Addict Behav 17(2):91-7, 2003.

Prochasca J.0.; Diclement, C.C. - Stages and process of self-change of smoking: toward an integrative model of change. J Consult Clin Psychol51(3):390-5, 1983.

RUMPF, H.J.; HAPKE, U.; MEYER, C.; JOHN, U. - Motivation to change drinking behavior: Comparison of alcohol-dependent individuals in a General Hospital and a general population sample. Gen Hospl Psych 21:348-53, 1999.

RYAN, R.M.; PLANT, R.W. - Initial motivations for alcohol treatment: relations with patient characteristics, treatment involvement and dropout. Addict Behav 20(3):279-97, 1995.

Share, D.; Mccrady, B.; Epstein, E. - Stage of change and decisional balance for women seeking alcohol treatment. Addict Behav 29:525-35, 2002.

Silva, E.A.; Ferri, C.P.; Formigonı, M.L.O.S. - Situações de recaída em pacientes durante o tratamento: Um estudo preliminar. J Bras Psiq 44(6):311-5, 1995.

Velasquez, M.M., Carbonari, J.P., Diclemente, C.C. - Psychiatric severity and behavior change in alcoholism: The Relation of the Transteoretical Model Variables to psychiatric distress in dually diagnosed patients. Addict Behav 24(4):481-96,1999. 